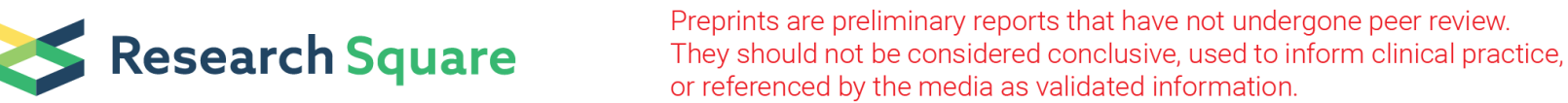

\section{Angiotensin 1 Peptide Conjugated CdSe/ZnS Quantum Dots Induce Cardiac-Specific Hydrogen Sulfide Production to Mitigate Myocardial Ischemia- Reperfusion Injury}

\section{Qing Wang}

Shanghai Jiao Tong University School of Medicine Affiliated Renji Hospital

Jie Min

980 Hospital

Xiaofei Xue

Changzheng Hospital: Shanghai Changzheng Hospital

Yue Yu

Shanghai Changzheng Hospital

Pei Wang

Shanghai Changzheng Hospital

Jun Wang

Shanghai Changzheng Hospital

Qixia Jiang

Shanghai Jiaotong University

Pengfei Zhang

Shanghai Skin Diseases Hospital

Jian Xiao ( $\square$ drxiaojian1980@126.com )

Changzheng Hospital: Shanghai Changzheng Hospital

\section{Research}

Keywords: nanocarrier, CdSe/ZnS QDs, cystathionine-y-lyase, angiotensin 1, endoplasmic reticulum stress, mitophagy

Posted Date: February 16th, 2021

DOl: https://doi.org/10.21203/rs.3.rs-201476/v1

License: (c) (1) This work is licensed under a Creative Commons Attribution 4.0 International License. Read Full License 


\section{Abstract}

Hydrogen sulfide $\left(\mathrm{H}_{2} \mathrm{~S}\right)$ is protective in myocardial ischemia/reperfusion injury (I/R). However, venous injection of NaHS and local injection of adenovirus with cystathionine-y-lyase (CSE) plasmids was used to increase $\mathrm{H}_{2} \mathrm{~S}$ concentration presented low-efficiency and side-effect. In this study, we explored a cardiac-specific approach to increase the local expression of $\mathrm{H}_{2} \mathrm{~S}$, the efficacy in $\mathrm{I} / \mathrm{R}$, and the underlying mechanism. Angiotensin 1 (AT1) conjugated nanocarrier (CdSe/ZnS quantum dots) carrying cystathionine-y-lyase (CSE) plasmid targeted myocardium was constructed and injected intravenously into the I/R animal model. The nanocarrier location was confirmed. The expression levels of CSE in different organs was also compared. The efficacy of nanocarrier was investigated in vivo and in vitro. In vivo fluorescence imaging system showed that the nanocarrier mostly enriched in the heart. Western blot demonstrated that CSE expression in the heart was higher in the AT1 group than the control group. There was no difference in other organs. Experiments in vitro showed that the nanocarrier had a high transfection efficiency. The CSE expression was also increased in the CSE group than the vector group. The IR rat was injected with the nanocarrier which decreased the infarct size and increased the ejection fraction. Cell viability and LDH concentration were also reduced in vitro at the same time. Key markers of endoplasmic reticulum stress (ERS) including CHOP, GRP78, elF2a expression level and mitophagy (Parkin, NIX, ATG) were all decreased in the CSE group. The rat was injected with an adenovirus vector carrying the CHOP gene which reversed the reduced mitophagy by CSE. In summary, the nanocarrier carrying CSE plasmid targeted myocardium with AT1 peptide can reduce the I/R without affecting other tissue. It inhibits ERS and mitophagy via the CHOP/GRP78/elF2a signaling pathway.

\section{Background}

Recent years have witnessed substantial progress in the prevention and treatment of acute myocardial infarction (AMI). The reperfusion strategy remains the current standard therapy. It includes percutaneous coronary intervention, coronary artery bypass graft surgery and thrombolytic therapy(1). However, myocardial ischemia/reperfusion injury (I/R) limits the efficacy of reperfusion treatment in AMI. I/R acts as a form of reperfusion-induced arrhythmias, myocardial stunning, microvascular obstruction and lethal myocardial reperfusion injury(2). Experimental studies have presented adequate protection for $\mathrm{l} / \mathrm{R}$ in animal models but the clinical translations are less satisfying(3). The urgent mission for $\mathrm{I} / \mathrm{R}$ research is to find a translational way for clinical application(4).

Hydrogen sulfide $\left(\mathrm{H}_{2} \mathrm{~S}\right)$ is an endogenous gasotransmitter which plays a vital role in the homeostasis of the cardiovascular system (5). Studies have shown that $\mathrm{H}_{2} \mathrm{~S}$ has a protective function in $\mathrm{I} / \mathrm{R}(6)$. Cystathionine- $\beta$-synthase (CBS), cystathionine-y-lyase (CSE/CGL) and 3-mercaptopyruvate sulfurtransferase (3-MST) in mammalian cells produces $\mathrm{H}_{2} \mathrm{~S}$. Overexpression of cardiac-specific CSE increases the production of endogenous $\mathrm{H}_{2} \mathrm{~S}$ which reduces infarct size and improved cardiac function(7). Research shows that $\mathrm{H}_{2} \mathrm{~S}$ plays a myocardial protective role against IR injury by regulating autophagy via mTOR activation(8). There are $\mathrm{H}_{2} \mathrm{~S}$ functions against the apoptosis of cardiomyocytes 
during the IR injury via the miR-1-HDAC4 signaling pathway(9). The clinical application of exogenous $\mathrm{H}_{2} \mathrm{~S}$ faces many problems. The $\mathrm{H}_{2} \mathrm{~S}$ unwanted side effect of systematic delivery include acute change of blood pressure, central neurotoxicity and respiratory depression(10).

The $\mathrm{H}_{2} \mathrm{~S}$ local delivery or local overexpression of CSE in the heart reduces the side effects but improves efficacy. Nanoparticle or nanocarrier has been developed as a novel delivery system which transports genes, drugs and other therapeutic agents with cellular targeting ability(11). Researchers have combined the nanocarrier and RNA interference (RNAi)-based therapy to enhance cellular uptake, effectiveness, targeted delivery and limited toxicity(11). A cardiac-specific nanoparticle delivery system containing CSE DNA plasmid was constructed for the study. The efficiency of the delivery system, the efficacy of the CSE overexpression, the protective effect in $\mathrm{I} / \mathrm{R}$ and the underlying mechanism were also evaluated.

\section{Results}

Preparation and characterization of PPQDs

As shown in Figure 1A, the oil-soluble QDs were transferred to the water phase using sulfhydrylcontaining glutathione molecules. The QDs modified by the water phase had a good uniform dispersion. The average particle size was about 7-10nm. The surfaces of QDs were modified with PEG to further improve the water solubility of QDs. The carboxyl group on the surface of QDs was covalently coupled with the amino group on the terminal lysine of the targeting peptide through EDC activation. A targeting peptide modified water-soluble QDs was thus obtained. As shown in Figure 1C, the agarose gel electrophoresis of QDs with different surface properties showed that the glutathione-modified QDs had the farthest migration distance and no obvious tailing. This indicated that glutathione modified QDs have good particle size and charge uniformity. The electrophoretic bands of QDs became more diffuse after modifying PEG. The migration distance was reduced. All of this indicated that PEG had been modified on the surface of QDs. However, PEG had some influence on the hydrated particle size distribution of QDs. After further modification of the targeting peptide, the electrophoretic bands of the QDs became wider. This indicated that the targeting peptide can be combined with the PEG-modified QDs. Also, it caused aggregation of some QDs.

The effect of the PEI/QD ratio on the accumulation of QDs was first studied after obtaining the QDs modified by the targeting peptide. As shown in Figure 1D, when the molar ratio of PEI/QD was 0.2, almost all the QDs were blocked in the electrophoresis loading hole. This demonstrated that the PEI amount at this ratio was sufficient to agglomerate the QDs through electrostatic self-assembly. Finally, a PEI/QD molar ratio of 0.3 was chosen to make PEl a proper excess and prepare DNA carrier clusters. The ratio of PEI-QD/DNA was further studied in order to optimize the embedding conditions of plasmids. The number of DNA plasmids were fixed and adjusted the number of PEI-QD. As shown in Figure 1E, when the molar ratio of PEI-QD/DNA was 0.5 , most of the plasmids were wrapped in QD clusters.

PPQDs specific enrichment and CSE expression in vivo 
I/R models in rats were established via ligation and release of the left anterior descending coronary artery (LAD) (ligation for 30 minutes, reperfusion for 24 hours). Using nanocarriers' fluorescence characteristics, the fluorescence imaging system (absorption wavelength of $460 \mathrm{~nm}$ ) in vivo showed that, the nanocarrier group's local myocardium fluorescence carrier AT1 peptide was significantly stronger than that of the empty nanocarrier group (Figure 2A).

I/R rat model was established. CSE nanocarriers with AT1 (AT1 group) and CSE nanocarriers without AT1 (control group) were injected through the internal jugular vein before reperfusion. The rat myocardium, skeletal muscle, liver and lung tissues were collected. The CSE expression levels in each tissue of IR rats were compared using Western-blot method. The results showed that the expression of CSE protein in the myocardial tissue of the AT1 group (nanocarriers with AT1 and CSE plasmids) was higher than that of the control group. There was no significant difference in the expression of CSE protein between the AT1 and control groups in skeletal muscle, liver and lung tissues. This indicated that the nanocarriers established could target the ischemic myocardium (Figure 2B).

Transfection of CSE nanocarriers and CSE expression in vitro

Neonatal rat cardiomyocytes were isolated and cultured in vitro. The standard medium was replaced with $50 \mu \mathrm{l} \mathrm{CSE} \mathrm{nanocarriers} \mathrm{(DNA} \mathrm{plasmids} \mathrm{in} \mathrm{PPQDs)} \mathrm{medium} \mathrm{and} \mathrm{observed} \mathrm{under} \mathrm{a} \mathrm{fluorescence}$ microscope. As shown in Figure 2C, the addition of PPQDs had no significant effect on cardiomyocytes' morphology and state. Figure $2 \mathrm{C}$ showed that CSE nanocarriers were red fluorescent and widely distributed in cardiomyocytesn indicating that PPQDs containing DNA plasmids have high transfection efficiency for cardiomyocytes. CSE nanomaterials were added to the cardiomyocytes. Thirty hours later the cardiomyocytes were collected and lysed while CSE expression was detected using WB. As shown in Figure 2D-2E, the CSE nanocarrier group had a significantly higher CSE expression level than the vector group.

Protective effect of CSE nanocarriers for I/R model in vitro and $\mathrm{H} / \mathrm{R}$ model in vivo

Whether CSE nanocarriers could protect rat from I/R injury in vivo was further explored. TTC results showed that the infarct area of mice in the CSE group was lower than that in the control group $(P<0.01$, Figure $3 \mathrm{~A}$ and $3 \mathrm{~B}$ ). This suggested that CSE nanocarriers had a myocardial protective effect in vivo. Echocardiography showed that the CSE nanocarriers increased the ejection fraction after I/R (Pख0.05, Figure $3 \mathrm{C}$ and $3 \mathrm{D})$.

CCK8 kit was used to detect the viability of cardiomyocytes after H/R. The results showed that the vitality of cardiomyocytes was decreased after H/R injury. However, cardiomyocytes' vitality in the CSE group was higher than that of the control group. This suggested that CSE nanocarriers could protect the cardiomyocytes in vitro $(\mathrm{P}<0.01$, Figure 3E). The ATP detection kit was applied to detect the effect of CSE nanocarriers on ATP content. It indicated that the ATP content of cardiomyocytes decreased after $\mathrm{H} / \mathrm{R}$ injury. However, the CSE group's ATP content was higher than that in the control group $(P<0.01$, Figure 3F). This indicated that CSE nanocarriers had a protective effect on mitochondrial function. The LDH 
content in the CSE group's cell culture medium was lower than that of the control group $(P<0.01$, Figure $3 G)$.

Inhibition of apoptosis by CSE nanocarriers in I/R model

As shown in Figure 4, the apoptosis rate of different groups was examined which showed that apoptosis was highly inhibited in the CSE group than the I/R group.

Inhibition of endoplasmic reticulum stress (ERS)-mitophagy- by CSE nanocarriers in I/R mode

Mitophagy and endoplasmic reticulum stress (ERS) play an essential role in myocardial ischemiareperfusion injury. The effect of CSE nanocarriers on mitophagy and ERS were also tested. After adding CSE nanocarriers, ERS-related protein expressions decreased including CHOP, GRP78 and elF2a. Furthermore, CSE nanocarriers affected mitophagy-related proteins. CSE nanocarriers inhibited the expression of Parkin, NIX and ATG proteins in the H/R model. CSE nanocarriers reduced excessive mitophagy and played a role in myocardial protection (Figure 5)

The CHOP adenovirus vector was constructed. The adenovirus carrying the $\mathrm{CHOP}$ gene was given 24 hours before the modeling of the isolated cardiomyocyte HR model. The H/R cell model was divided into four groups: normal control group, HR group, HR+CSE group and Ad-CHOP+HR+CSE group. CHOP overexpression attenuated the inhibitory effect of CSE on mitophagy (Figure 6).

\section{Discussion}

Nanomedicines have a significant development in clinics especially the nanoparticles. Nanocarriers are widely applied in the delivery of genes, drugs and other therapeutic agents with cellular targeting ability. Nanomedicine gained its popularity because its ability to reduce toxicity, by modulating the distribution of drugs and enriches the target sites with chemotherapeutic drugs or recombinant DNA and RNAi molecules(12). This study developed a nanocarrier system of PPQDs carrying CSE DNA plasmid which specifically targeted the heart's I/R area and presented with satisfying target characterization and protective efficacy.

Different types of nanoparticles have been developed as nanocarriers including the organic nanoparticles and inorganic nanoparticles. As a new inorganic/non-metallic nanoparticle, QDs have attracted adequate attention. This is because QDs have advantages of good photostability, vigorous fluorescence intensity and various emission wavelengths(13). In the study, the CdSe/ZnS QDs was chosen as the nanocarriers which is one of the most commonly used QDs. Liu et al. applied CdSe/ZnS QDs to label mesenchymal stem cells (MSCs). CdSe/ZnS-labeled MSCs targeted pancreas tissues in diabetic rats in vivo and significantly reduced the blood glucose levels in diabetic rats(14). However, the CdSe/ZnS QDs exhibited toxicity on the heart, liver, kidney, immune system and reproductive system(15). CdSe/ZnS QDs are encapsulated with PEG and conjugated with specific peptide to achieve organ-targeted function. Lei and colleagues conjugated Tat peptide with PEG encapsulated CdSe/ZnS QDs and were introduced into living 
mesenchymal stem cells. Characteristic fluorescence of QDs was observed primarily in the liver, the lung and the spleen(16). Little or no QDs accumulated in the brain, the heart or the kidney(16). Findings demonstrated that, the conjugation of peptide and PPQDs can enrich a nanocarrier in a specific organ. AT1 was used as the target peptide. Studies found that the AT1 target the heart and AT1 receptor expression was high in the I/R model. QDs with targeting peptide transport plasmids to ischemic cardiomyocytes while carrying the fluorescence were used to track the whereabouts of nanoclusters. The plasmids were successfully transfected into the cardiomyocytes and increased the protein expression of CSE. The best ratio of the PEI/QD and PEI-QD/DNA were tested in order to achieve a satisfying transfection efficiency.

The CSE expression was increased in the myocardium without changes in muscle, liver and lung after intravenous injection of the nanocarriers containing CSE plasmids. A study has shown that CSE's overexpression increases the endogenous production of $\mathrm{H}_{2} \mathrm{~S}$ which protects the myocardium(17). However, traditional transfection of CSE overexpression in transgenic mice DNA plasmids led to circulating production of $\mathrm{H}_{2} \mathrm{~S}$ and augmented endothelial-dependent vasorelaxation response in thoracic aorta(18). The global effect could possibly cause unwanted side effect. What's more, intravenous injection of CSE DNA plasmids had the disadvantage of low transfection efficiency, while local injection in myocardium were confronted with trauma and low translational value. In this study, the efficacy of the nanocarriers on $\mathrm{I} / \mathrm{R}$ was further validated. The administration of PPQDs mitigate myocardial injury and preserve the cardiac function. The potential mechanism underlying the protective role in apoptosis, mitochondrial function and mitophagy was investigated. The ATP content and mitochondrial function of the CSE group were higher than the control group. Elrod also studied the potential of $\mathrm{H}_{2} \mathrm{~S}$ as a cardioprotective agent. Elrod found that the $\mathrm{H}_{2} \mathrm{~S}$ protective effect was associated with inhibition of myocardial inflammation and preservation of both mitochondrial structure and function after $1 / R(7)$. The study examined the apoptosis rates of different groups using TUNNEL staining. It showed that the CSE group had decreased apoptosis rate. The role of apoptosis in I/R was studied before and demonstrated that $\mathrm{H}_{2} \mathrm{~S}$ protected the cardiomyocytes against IR-induced apoptosis by stimulating Bcl-2(19). Studies also have showed a tight connection and mutual relationship between apoptosis and ERS in $\mathrm{I} / \mathrm{R}(20)$. $\mathrm{Li}$ found that dexmedetomidine attenuates I/R in diabetes mellitus by inhibiting ERS(20, 21).

Furthermore, Zhang and colleagues confirmed that OPA1-related mitochondrial fusion/mitophagy was modulated by melatonin in cardiac I/R injury. The potential role of mitophagy and ERS in CSE's protective role in I/R were investigated. The ERS and mitophagy signaling pathway was activated after the H/R process. However, when administered with nanocarriers carrying CSE plasmids, the signaling pathway was hindered and down-regulated. The adenovirus with the CHOP gene was applied and reversed CSE inhibition. The findings above supported that CSE nanocarriers protect the heart in I/R mainly through apoptosis inhibition from ERS and mitophagy signaling pathway.

\section{Conclusions}


In summary, a myocardium-specific nanocarrier system was constructed. When carrying the CSE plasmids, the PPQDs transfect the cardiomyocytes and increase the CSE expression. Therefore, CSE nanocarriers mitigate the $\mathrm{I} / \mathrm{R}$ and preserve the cardiac function by reducing apoptosis via inhibition of the ERS and mitophagy signaling pathway.

\section{Material And Methods}

Synthesis and Characterization of CdSe/ZnS quantum dots and Oil-soluble CdSe/ZnS core-shell quantum dots (QDs) were purchased from the Suzhou Xingshuo Nanotechnology Company. The QDs were phase transferred using glutathione (GSH) and cross-linked with carboxyl-PEG-carboxyl groups (Sigma-Aldrich CAT\#14565, Mn = 2000) to obtain PEGylated polymer-encapsulated QDs. One $\mathrm{mL}$ of CdSe/ZnS QDs (10 mg) chloroform solution was added to $10 \mathrm{~mL}$ of $250 \mathrm{mg}$ GSH plus $200 \mathrm{mg}$ sodium hydroxide aqueous solution which were magnetically stirred. The chloroform in the mixture gradually evaporated at room temperature. The ligand exchange of QDs is also completed at the water-oil interface. The QDs aqueous solution was dialyzed against borate buffer $(\mathrm{pH} 8.0,20 \mathrm{mM})$ for at least 72 hours to remove free glutathione in the solution. The glutathione molecules on the QD surface are cross-linked through carboxyl-PEG-carboxyl which helps to improve colloidal stability. The carboxyl group in the PEG crosslinker is activated by the activator 1-(3-dimethylaminopropyl) 3-ethyl carbodiimide hydrochloride (EDC) $(10 \mathrm{mg} / \mathrm{mL})$. After 15 minutes of activation at room temperature, the active PEG crosslinker was added to the GSH-QDs aqueous solution (where the molar ratio of QD/PEG is 1/1000) under magnetic stirring at room temperature for 2 hours. The resulting PEGylated QDs solution was dialyzed in borate buffer for 72 hours to remove unbound PEG and small molecules. Finally, the QDs solution was concentrated by rotary evaporation to about $5 \mu \mathrm{M}$ for later use.

Modification of peptides

Gly-Gly-Gly-Gly-Asp-Arg-Val-Tyr-lle-His-Pro-Phe is a peptide targeting ischemic myocardium with lysine residues at the end which was synthesized by GenScript. The purity of peptide detected using highpressure liquid chromatography was $>95 \%$. The target peptide was covalently coupled to the carboxyl group on the surface of the QDs through the terminal amino group. In the general coupling process, the PEGylated QDs were dispersed in phosphate buffer ( $\mathrm{pH} 6.0,25 \mathrm{mM})$ and EDC was added (Concentration: about $1 \mathrm{mg} / \mathrm{mL}$ ). The reaction was activated at room temperature for 0.5 hour. The excess activator was removed using ultrafiltration and the targeting peptide (concentration about $1 \mathrm{mg} / \mathrm{mL}$ ) was added and reacted at room temperature for 1 hour. The excess targeting peptide was removed using ultrafiltration to obtain the target. QDs was coupled to peptides (Peptide-PEG-QDs, PPQD).

\section{Package of DNA Plasmids}

Polyvinylimide (Mw 2000) was dispersed in PB buffer $(1 \mathrm{mg} / \mathrm{mL})$. The $\mathrm{pH}$ was adjusted to neutral. The targeted peptide-coupled QDs (PPQDs) were mixed with different proportions of polyvinylimide and quickly Stirred. Moreover, $1 \%$ agarose gel electrophoresis was performed to analyze the electrostatic selfassembly process of QDs and PEl in order to get a better QD/PEl ratio. Under a better QD/PEI ratio, the 
plasmids with different DNA concentrations were mixed with QD/PEl. Agarose gel electrophoresis was used to analyze the plasmids better ratio loaded on the QDs (Agarose concentration 1\%, voltage $80 \mathrm{~V}$, time 15 minutes).

\section{Animals}

Male SPF grade Sprague-Dwaly (SD) rats (weighing 250-300g) and c57BL6/J male mice (weighing 18$22 \mathrm{~g}$ ) were purchased from the Second Military Medical University Experimental Animal Center. The Animal Research Ethics Committee approved the animal protocols of the Second Military Medical University in accordance with the Care and Use of Laboratory Animals published by the US National Institutes of Health (NIH publication vol. 25 no. 28, revised 1996). The experimental animals were kept under standard room conditions with a temperature of $25 \pm 1^{\circ} \mathrm{C}$, the humidity of $60 \%$ and 6 a.m. to 6 p.m. natural lights. Standard rodent chow and water were given routinely.

The experimental animals were divided into the sham group, the IR group and the CSE group. The IR treatment was induced by ligating the left anterior descending artery (LAD) for 30 minutes and reperfusion for 24 hours as previously described(8). The sham group underwent thoracotomy without ligation. The IR group were subjected to IR treatment and intravenous injection with $20 \mu$ vector nanocarriers before reperfusion. The CSE group were subjected to IR treatment and intravenous injection with $20 \mu \mathrm{l} \mathrm{CSE} \mathrm{nanocarrier} \mathrm{before} \mathrm{reperfusion.}$

In vivo fluorescence imaging system

The Bruker in-vivo F PRO system was applied in vivo whole-animal imaging analysis. Images of the postinjection of CSE nanocarriers were captured and analyzed. The excitation and emission filters were set to 410 and $700 \mathrm{~nm}$ (bandpass, $\pm 15 \mathrm{~nm}$ ) respectively.

\section{Echocardiography}

Echocardiography was performed blindly four days after the surgery (VisualSonics Vevo 2100, M-mode, and $30 \mathrm{MHz}$ probe). The conscious mice were examined at the mid-papillary level. LV dimensions in diastole and systole especially the ejection fraction (EF) were measured following the standard procedures and calculations.

Infarct size measurement

Evans blue dye (2\%, Solarbio, China) was injected in the vein but the area at risk (AAR) remained nondyed (17). The heart's infarct size was assessed using the TTC-staining technique and digital measurement with Image-Pro Plus software (Media Cybernetics). The area at risk/left ventricle ratio (AAR/LV) and scar area/left ventricle ratio was quantified and calculated.

Apoptosis 
TUNEL staining was conducted as previously described(20). Image-Pro Plus software (Media Cybernetics) was used to detect the apoptosis rates of TUNEL sections.

\section{Cell culture and treatment}

The neonatal cardiomyocytes of rats were cultured and used to conduct the hypoxia-reoxygenation $(H / R)$ experiments ${ }^{[9]}$. The cardiomyocytes were divided into four groups. For the $\mathrm{N}+\mathrm{Vector}$ group, the cells were cultured in DMEM with $50 \mu$ vector nanocarriers in $5 \% \mathrm{CO}_{2}$ and $95 \%$ air for 24 hours. Moreover, in the $\mathrm{N}+\mathrm{CSE}$ group the cells were cultured in DMEM with $50 \mu \mathrm{l}$ CSE nanocarriers in $5 \% \mathrm{CO}_{2}$ and $95 \%$ air for 24 hours. Additionally, for the HR+Vector group, the cells were cultured in DMEM with $50 \mu$ vector nanocarriers with $1 \% \mathrm{O}_{2}, 5 \% \mathrm{CO}_{2}$ and $94 \% \mathrm{~N}_{2}$ atmosphere for $24 \mathrm{~h}$ and $5 \% \mathrm{CO}_{2}$ and $95 \%$ air for 6 hours. lastly, for the HR+CSE group, the cells were cultured in DMEM with $50 \mu \mathrm{l} C S E$ nanocarriers with $1 \% \mathrm{O}_{2}, 5 \%$ $\mathrm{CO}_{2}$ and $94 \% \mathrm{~N} 2$ atmosphere for 24 hours and $5 \% \mathrm{CO}_{2}$ and $95 \%$ air for 6 hours.

ATP and LDH test

The conditioned cell culture supernatant $(200 \mu \mathrm{l})$ was collected after HR and was used to determine LDH levels using a spectrophotometric kit (Roche Diagnostics) and ATP concentrations using an ATP assay (Colorimetric/Fluorometric, Abcam, ab83355) according to the manufacturer's instructions.

Western blotting

Protein concentrations were determined using the BCA protein assay kit according to the manufacturer's protocol. Western blotting (WB) was conducted as previously described. Primary antibodies were listed as follows: CSE, Abcam, ab96755; CHOP, Cell Signaling Technology, 2895S; GRP78, Abcam, ab21685; elF2a, Abcam, ab169528; ATF-6, Cell Signaling Technology, \#65880; Parkin, Cell Signaling Technology, \#2132; ATG7, Cell Signaling Technology, \#2631; Nix, Abcam, ab109414; LC-3, Abcam, ab192890; GAPDH, ThermoFisher, MA5-15738-D680. The grayscale value of the bands was quantified using Image lab software (BIO-RAD, USA).

Statistical analysis

GraphPad Prism 7.0 was used to analyze the data in the study. The continuous data were expressed as the mean \pm standard error (SEM). One-way ANOVA was used followed by Post-hoc $t$ Turkey's test to compare the variables of different groups. A P-value $<0.05$ was considered to have statistical significance.

\section{Declarations}

\section{Acknowledgements}

None 


\section{Author's contributions}

Conceived and designed the experiments: PZ and JX. PPQDs preparation: PZ. Animal experiment: QW and XX. Molecular experiments: JM. Statistical analysis: PW, JW and YY. Manuscript redaction: QW and XX. Manuscript editing: QJ and JX. All authors read and approved the final manuscript.

\section{Funding}

This work was supported by the National Nature Science Foundation of China (NO. 82070255), military medical talent project (Yuanhang) of Naval Medical University, Pyramid talent project of Changzheng hospital, Cultivation of interdisciplinary projects区YG2021QN141区, Scientific research project of health and Health Committee of Changning District (20194Y003), Science and Technology Support Project of Chinese and Western Medicine (19401930600), Scientific Research Project of Shanghai Health and Family Planning Commission (201840023), and General Project of TCM Scientific Research Subject of Shanghai Health and Family Planning Commission(2018LP032).

\section{Availability of data and materials}

The datasets used and/or analysed during the current study are available from the corresponding author on reasonable request.

\section{Ethics approval and consent to participate}

The Animal Research Ethics Committee approved the animal protocols of the Second Military Medical University in accordance with the Care and Use of Laboratory Animals published by the US National Institutes of Health (NIH publication vol. 25 no. 28, revised 1996).

\section{Competing interests}

The authors declare that they have no competing interests.

\section{References}

1. Yellon DM, Hausenloy DJ. Myocardial reperfusion injury. The New England journal of medicine. 2007;357(11):1121-35.

2. Neri M, Riezzo I, Pascale N, Pomara C, Turillazzi E. Ischemia/Reperfusion Injury following Acute Myocardial Infarction: A Critical Issue for Clinicians and Forensic Pathologists. Mediators of inflammation. 2017;2017:7018393.

3. Binder A, Ali A, Chawla R, Aziz HA, Abbate A, Jovin IS. Myocardial protection from ischemiareperfusion injury post coronary revascularization. Expert review of cardiovascular therapy. 2015;13(9):1045-57.

4. Heusch G. Cardioprotection: chances and challenges of its translation to the clinic. Lancet (London, England). 2013;381(9861):166-75. 
5. Citi V, Piragine E, Testai L, Breschi MC, Calderone V, Martelli A. The Role of Hydrogen Sulfide and H2Sdonors in Myocardial Protection Against Ischemia/Reperfusion Injury. Current medicinal chemistry. 2018;25(34):4380-401.

6. Karwi QG, Bice JS, Baxter GF. Pre- and postconditioning the heart with hydrogen sulfide (H(2)S) against ischemia/reperfusion injury in vivo: a systematic review and meta-analysis. Basic research in cardiology. 2018;113(1):6.

7. Elrod JW, Calvert JW, Morrison J, Doeller JE, Kraus DW, Tao L, et al. Hydrogen sulfide attenuates myocardial ischemia-reperfusion injury by preservation of mitochondrial function. Proceedings of the National Academy of Sciences of the United States of America. 2007;104(39):15560-5.

8. Xiao J, Zhu X, Kang B, Xu J, Wu L, Hong J, et al. Hydrogen Sulfide Attenuates Myocardial HypoxiaReoxygenation Injury by Inhibiting Autophagy via mTOR Activation. Cellular physiology and biochemistry : international journal of experimental cellular physiology, biochemistry, and pharmacology. 2015;37(6):2444-53.

9. Kang B, Li W, Xi W, Yi Y, Ciren Y, Shen H, et al. Hydrogen Sulfide Protects Cardiomyocytes against Apoptosis in Ischemia/Reperfusion through MiR-1-Regulated Histone Deacetylase 4 Pathway. Cellular physiology and biochemistry : international journal of experimental cellular physiology, biochemistry, and pharmacology. 2017;41(1):10-21.

10. Almeida AF, Guidotti TL. Differential sensitivity of lung and brain to sulfide exposure: a peripheral mechanism for apnea. Toxicological sciences : an official journal of the Society of Toxicology. 1999;50(2):287-93.

11. Shen H, Huang X, Min J, Le S, Wang Q, Wang X, et al. Nanoparticle Delivery Systems for DNA/RNA and their Potential Applications in Nanomedicine. Current topics in medicinal chemistry. 2019;19(27):2507-23.

12. Garbayo E, Pascual-Gil S, Rodríguez-Nogales C, Saludas L, Estella-Hermoso de Mendoza A, BlancoPrieto MJ. Nanomedicine and drug delivery systems in cancer and regenerative medicine. Wiley interdisciplinary reviews Nanomedicine and nanobiotechnology. 2020;12(5):e1637.

13. Li L, Chen Y, Xu G, Liu D, Yang Z, Chen T, et al. In vivo Comparison of the Biodistribution and Toxicity of InP/ZnS Quantum Dots with Different Surface Modifications. International journal of nanomedicine. 2020;15:1951-65.

14. Liu H, Tang W, Li C, Lv P, Wang Z, Liu Y, et al. CdSe/ZnS Quantum Dots-Labeled Mesenchymal Stem Cells for Targeted Fluorescence Imaging of Pancreas Tissues and Therapy of Type 1 Diabetic Rats. Nanoscale research letters. 2015;10(1):959.

15. Li L, Tian J, Wang X, Xu G, Jiang W, Yang Z, et al. Cardiotoxicity of Intravenously Administered CdSe/ZnS Quantum Dots in BALB/c Mice. Frontiers in pharmacology. 2019;10:1179.

16. Lei Y, Tang H, Yao L, Yu R, Feng M, Zou B. Applications of mesenchymal stem cells labeled with Tat peptide conjugated quantum dots to cell tracking in mouse body. Bioconjugate chemistry. 2008;19(2):421-7. 
17. Hu X, Liu B, Wu P, Lang Y, Li T. LncRNA Oprm1 overexpression attenuates myocardial ischemia/reperfusion injury by increasing endogenous hydrogen sulfide via Oprm1/miR-30b-5p/CSE axis. Life sciences. 2020;254:117699.

18. Xia H, Li Z, Sharp TE, 3rd, Polhemus DJ, Carnal J, Moles KH, et al. Endothelial Cell Cystathionine YLyase Expression Level Modulates Exercise Capacity, Vascular Function, and Myocardial Ischemia Reperfusion Injury. Journal of the American Heart Association. 2020;9(19):e017544.

19. Kang B, Hong J, Xiao J, Zhu X, Ni X, Zhang Y, et al. Involvement of miR-1 in the protective effect of hydrogen sulfide against cardiomyocyte apoptosis induced by ischemia/reperfusion. Molecular biology reports. 2014;41(10):6845-53.

20. Li J, Zhao Y, Zhou N, Li L, Li K. Dexmedetomidine Attenuates Myocardial Ischemia-Reperfusion Injury in Diabetes Mellitus by Inhibiting Endoplasmic Reticulum Stress. Journal of diabetes research. 2019;2019:7869318.

21. Zhang BF, Jiang H, Chen J, Guo X, Li Y, Hu Q, et al. Nobiletin ameliorates myocardial ischemia and reperfusion injury by attenuating endoplasmic reticulum stress-associated apoptosis through regulation of the PI3K/AKT signal pathway. International immunopharmacology. 2019;73:98-107.

\section{Figures}



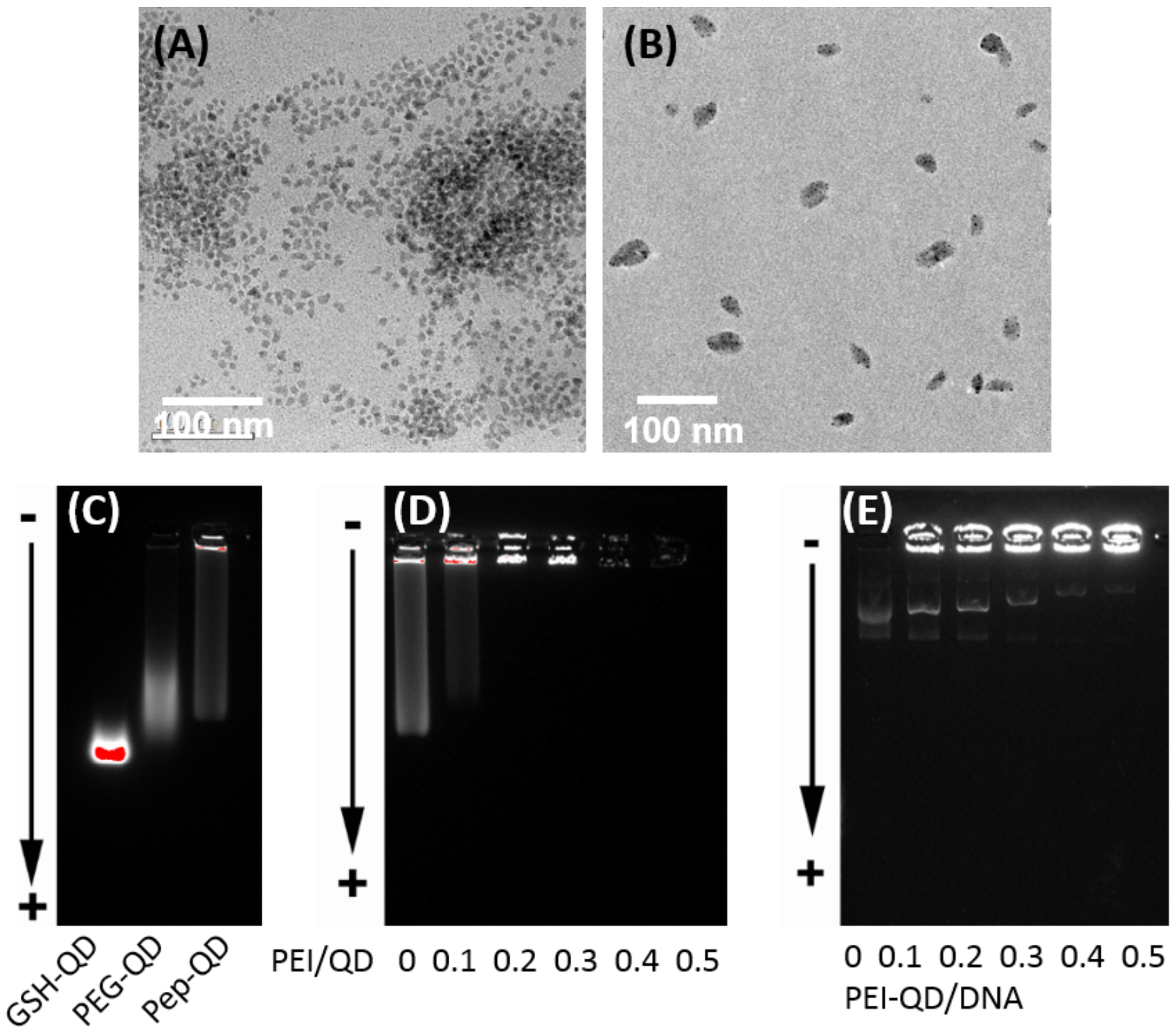

Figure 1

Characterizations of different CdSe/ZnS QDs. A, TEM graphs of glutathione-modified CdSe/ZnS QDs. B, TEM graphs of targeted quantum dot clusters loaded with DNA plasmids. C, Agarose gel electrophoresis diagram of QDs with different surface properties from left to right: glutathione, polyethylene glycol PEG, targeting peptide. D, Agarose gel electrophoresis diagram of different mixed ratios of PEI/QDs. E, Agarose gel electrophoresis diagram after mixing PEI-QD with different ratios of DNA plasmids. QDs, quantum dots. TEM, Transmission electron microscope. 
A

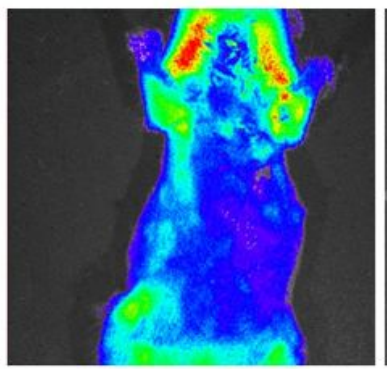

Control

B

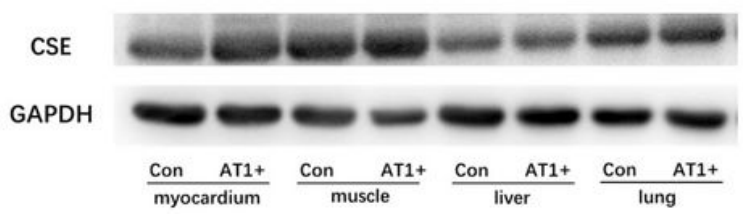

C

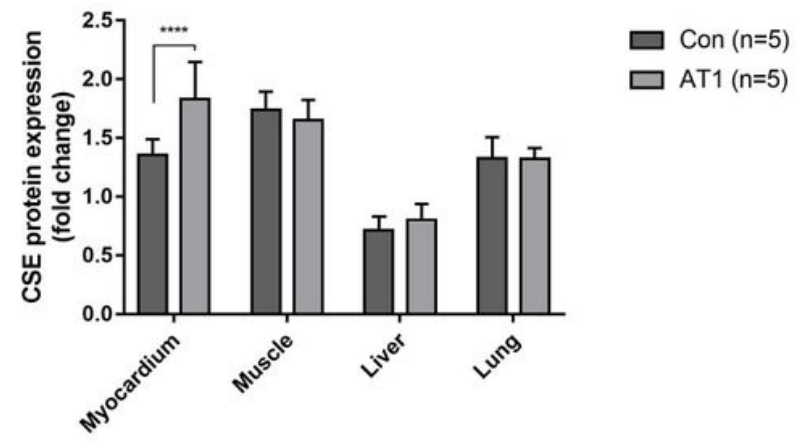

D

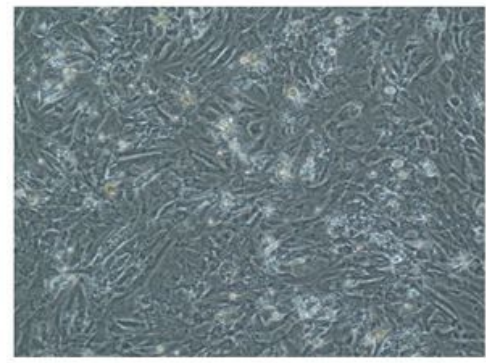

Bright field

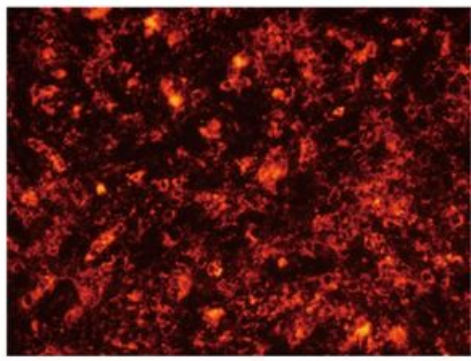

Fluorescence

$\mathrm{E}$

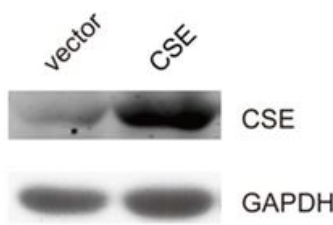

$\mathrm{F}$

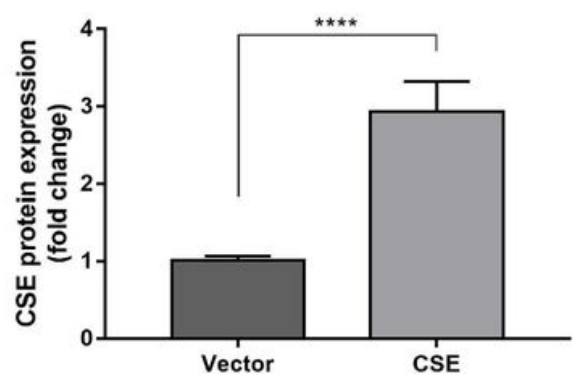

\section{Figure 2}

Nanocarrier targeted myocardium and increased CSE expression in vivo and in vitro. A, in vivo fluorescence imaging system showing nanocarriers conjugated with AT1 enriched in myocardium of rats. $B, C S E$ expression level of different tissues in rat injected with CSE nanocarriers. C, isolated cardiomyocytes transfected with CSE nanocarriers in bright field and fluorescence filed. D-E, CSE expression level in cells transfected with CSE plasmids and vector. Expressed as Mean \pm SEM. $\star \star \star \star P<0.0001$. CSE, cystathionine- $y$-lyase; AT1, Angiotensin 1. 
A
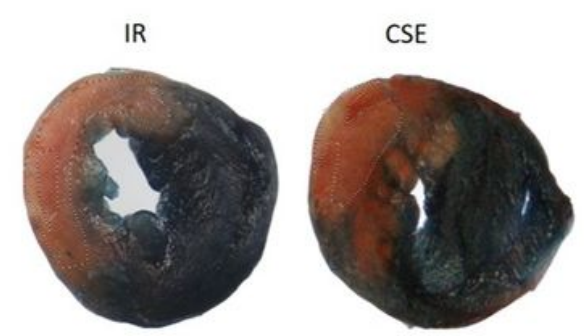

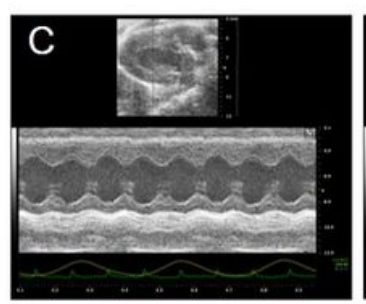

sham

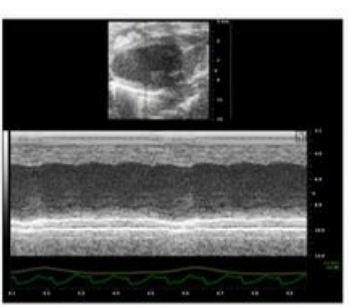

IR
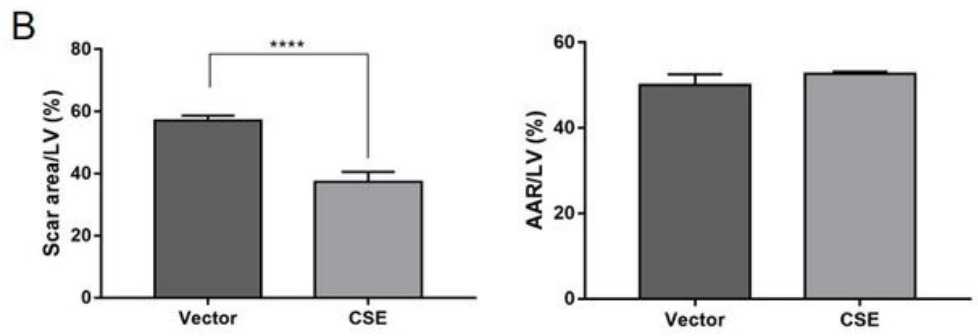

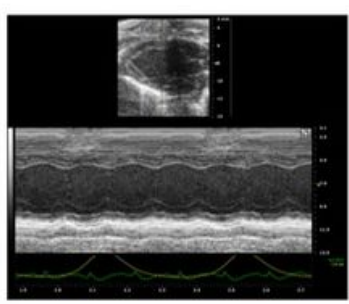

CSE
E

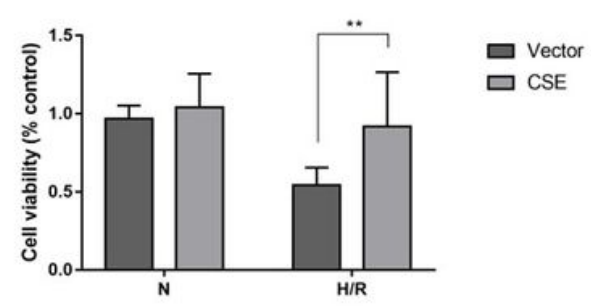

$\mathrm{F}$

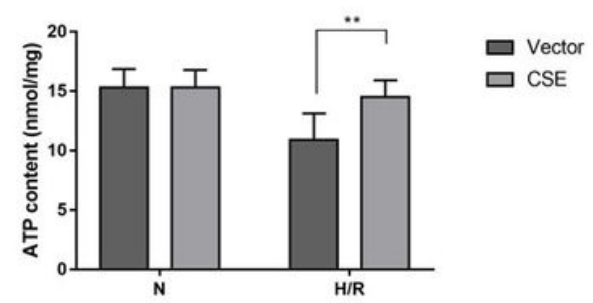

G
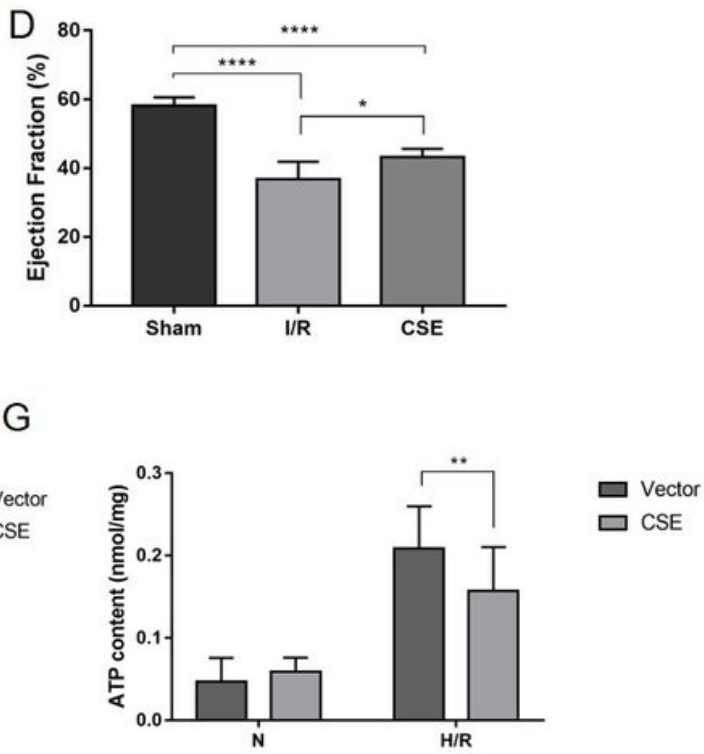

Figure 3

Nanocarrier mitigated I/R and preserved cardiac function. A, TTC/Evans blue staining showing myocardial infarction area in mice. B, quantitative indicators of area at risks/left ventricle and scar area/ left ventricle. C, the systolic function of different groups detected using echocardiography. D, ejection fraction of different groups. E, call viability of cardiomyocytes. F, ATP content of different groups. G, LDH activity of different groups. Expressed as Mean \pm SEM. ${ }^{*} P<0.05$, ${ }^{\star *} P<0.01$, ${ }^{\star \star *} * \mathrm{P}<0.0001$. I/R, myocardial ischemia/reperfusion injury. 

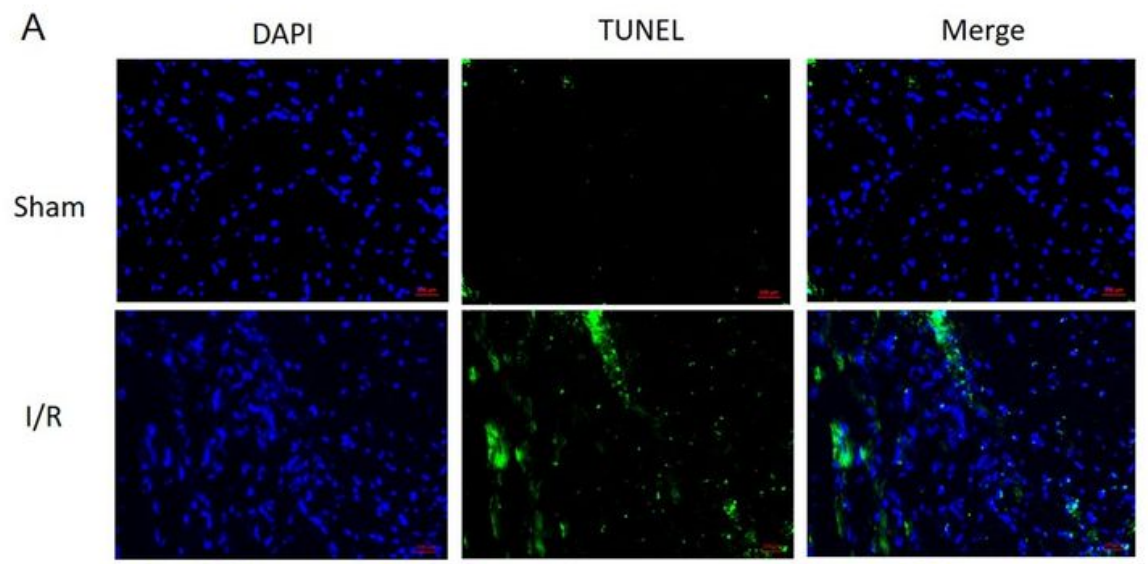

B
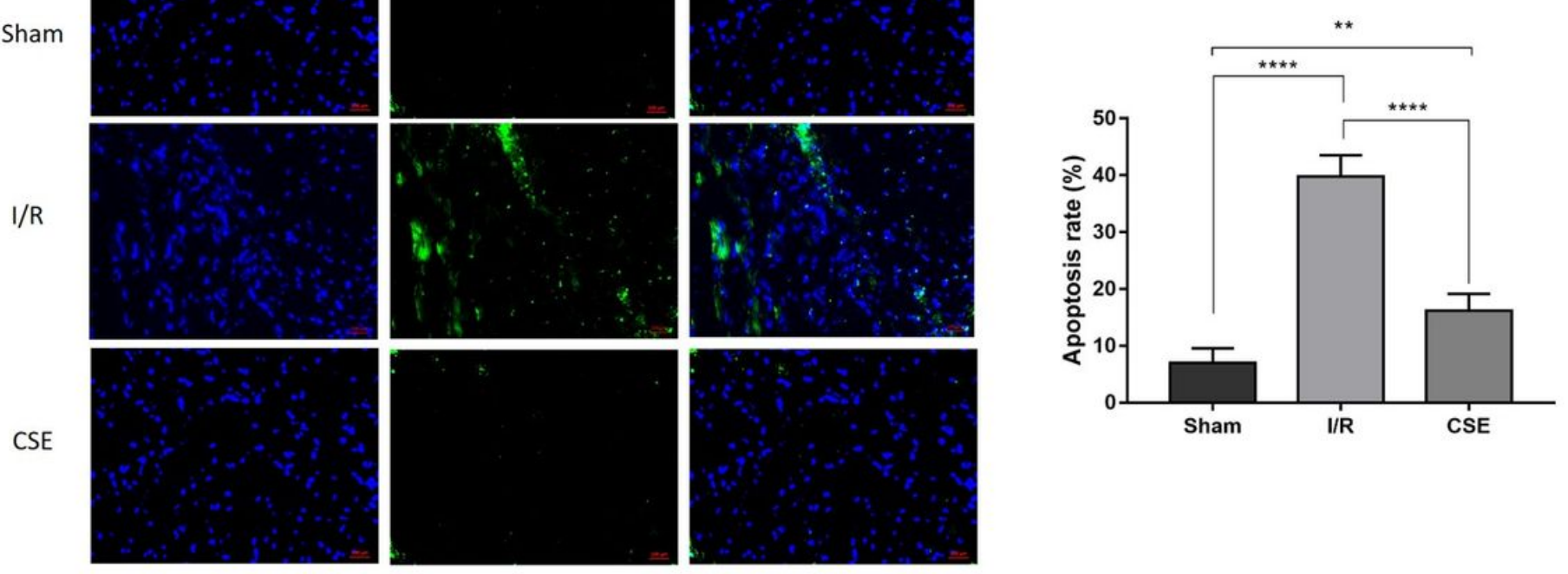

Figure 4

TUNEL staining of the apoptosis in the I/R model. A Representative section of TUNEL staining; $B$ Apoptosis rates of different groups. ${ }^{*} P<0.01,{ }^{*} \star * P<0.0001 .1 / R$, myocardial ischemia/reperfusion injury. 
A
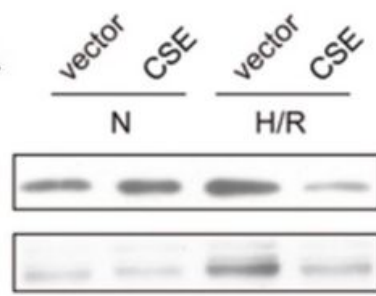

GRP78

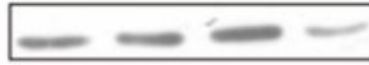

elF2a
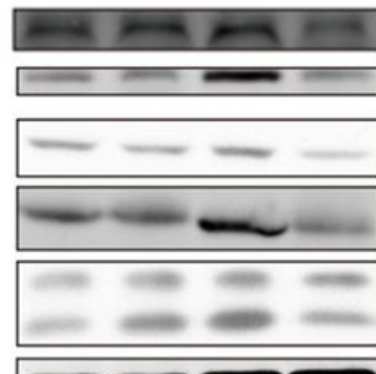

ATF6

Parkin

ATG7

Nix

LC-3

GAPDH

F

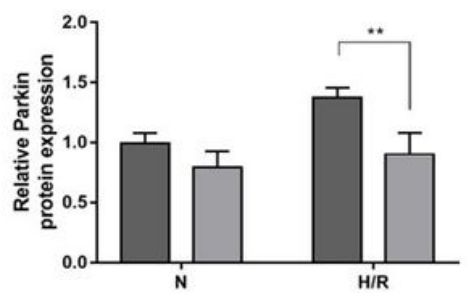

I

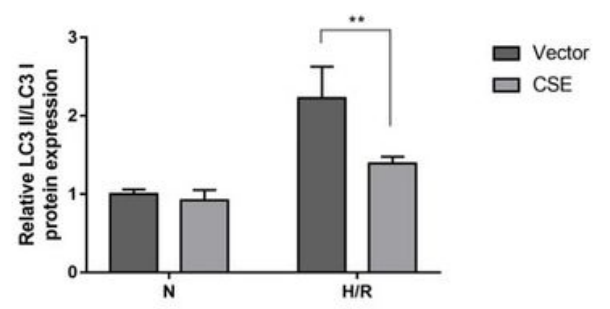

B

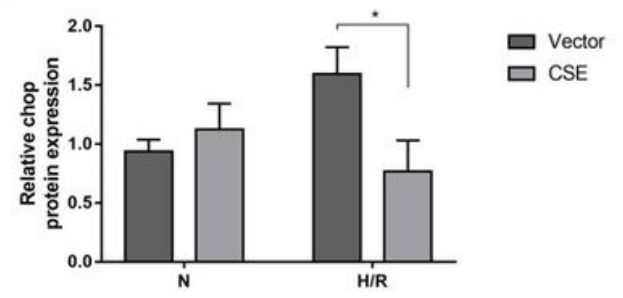

D

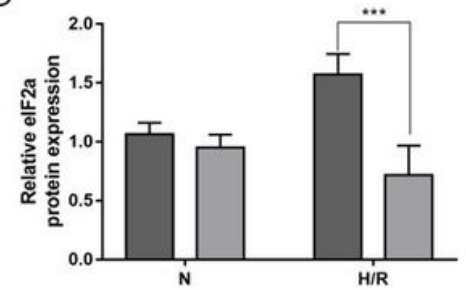

G
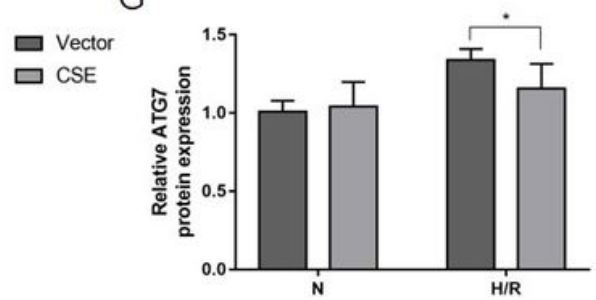

C

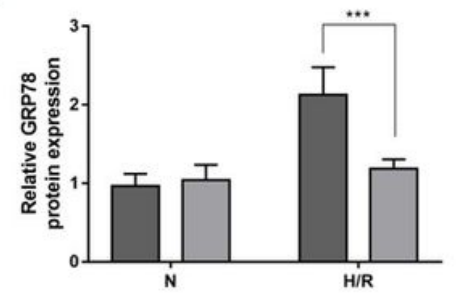

ㅁ Vector

口 CSE

F
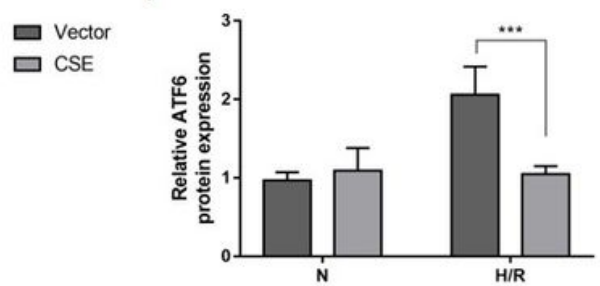

$\square$ Vector ㅁ CSE

$\mathrm{H}$

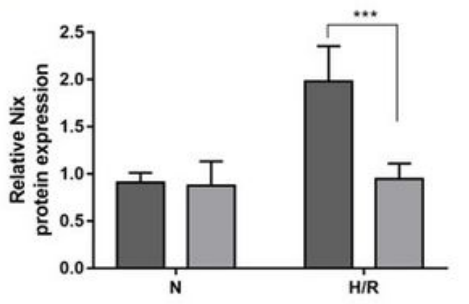

$\square$ Vector

므 Vector

口 CSE

Figure 5

CSE Nanocarriers inhibited the protein expression of ERS and mitophagy. A, representative WB bands of critical markers of ERS and mitophagy. B-I, quantitative indicators of critical markers of ERS and mitophagy in WB. Expressed as Mean \pm SEM. $n=5$ for each group, ${ }^{\star} P<0.05, * \star P<0.01,{ }^{*} * * P<0.001$. CSE, cystathionine-y-lyase; ERS, endoplasmic reticulum stress; WB, western blotting. 
A
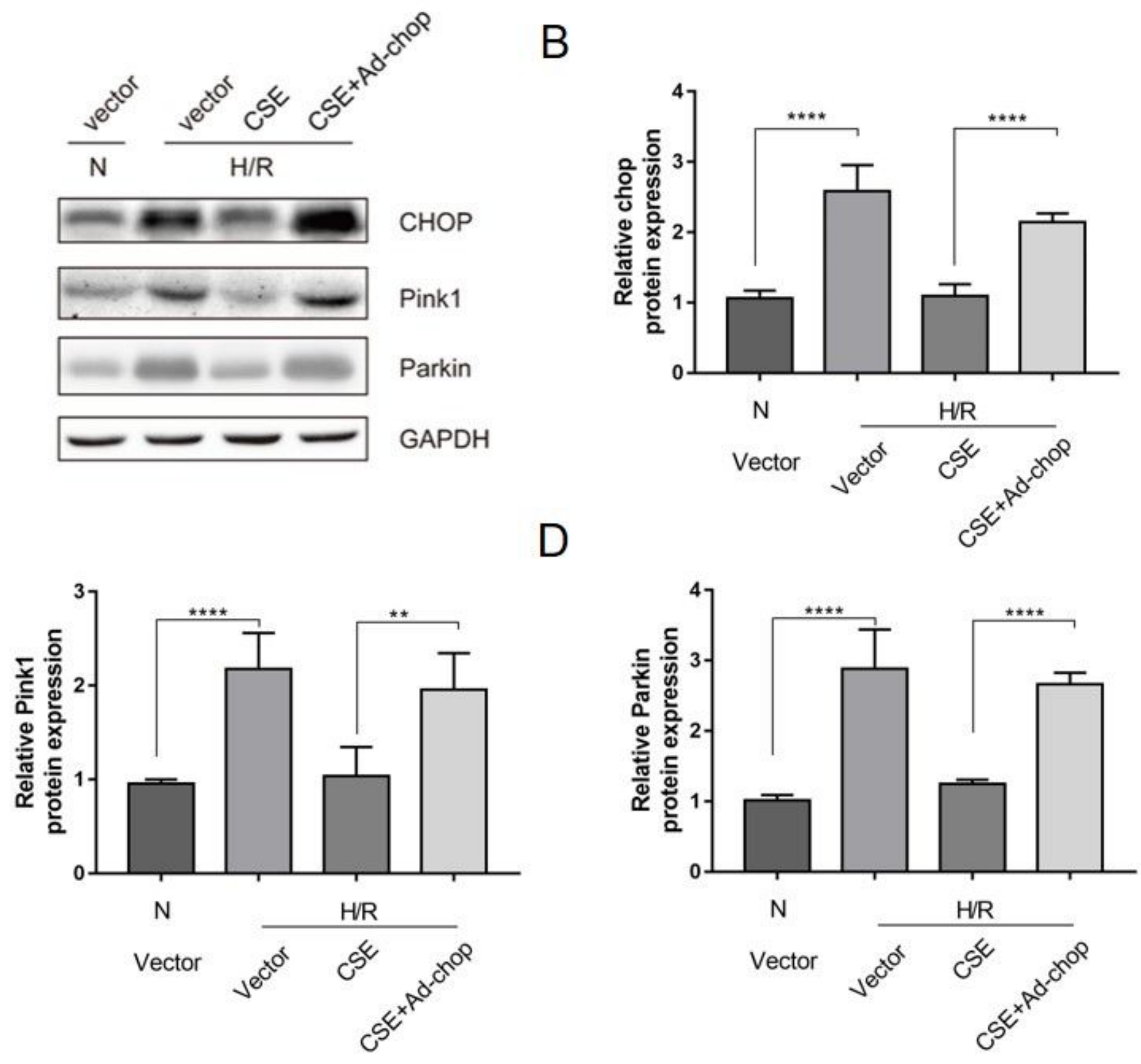

Figure 6

The effect of overexpression of CHOP on the inhibition of mitophagy in HR cardiomyocytes by CSE. A, representative WB bands. B-I, quantitative indicators of WB results. Expressed as Mean \pm SEM. $n=5$ for each group, ${ }^{* *} P<0.01,{ }^{* * * *} P<0.0001$. CSE, cystathionine-y-lyase; ERS, endoplasmic reticulum stress; WB, western blotting. 


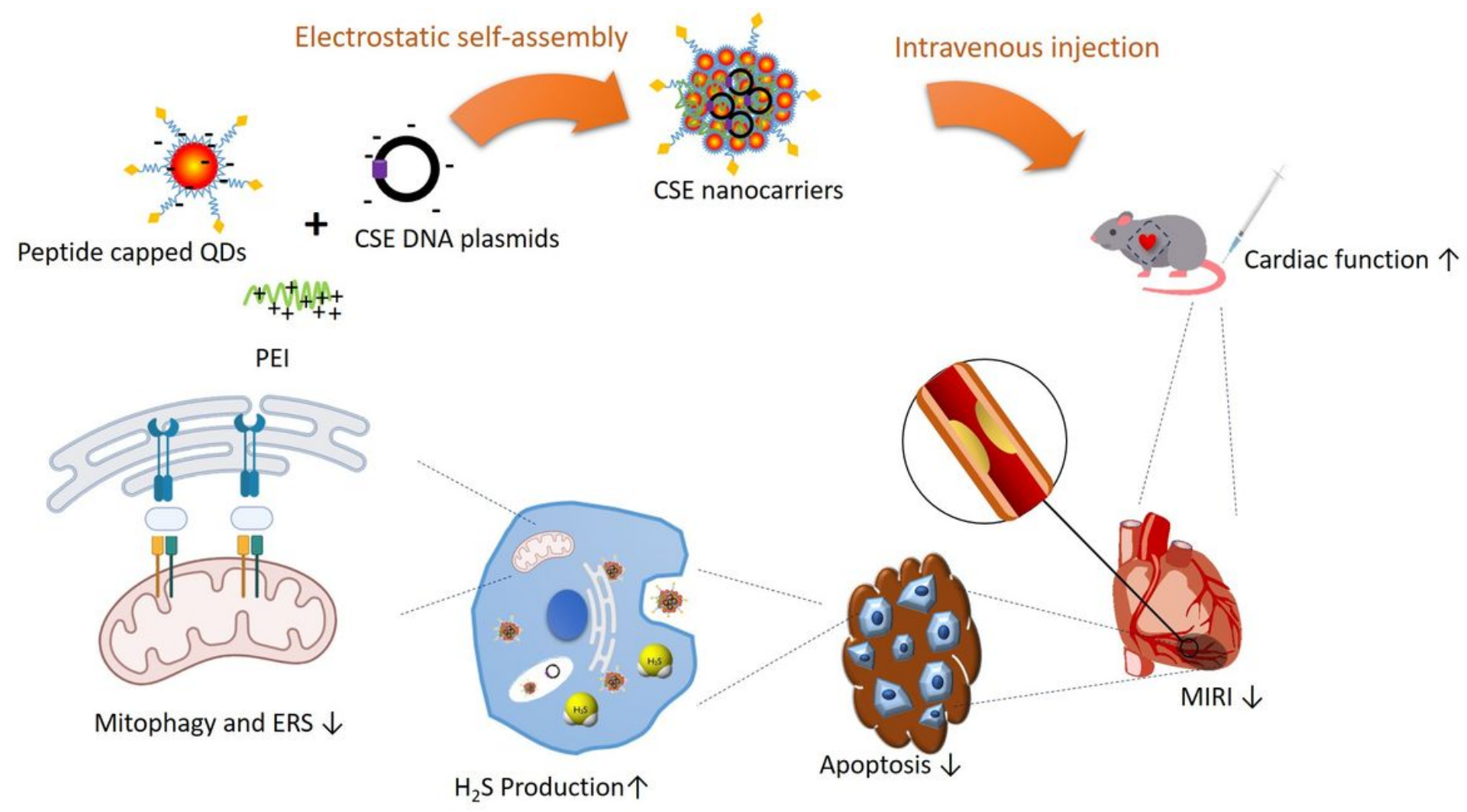

\section{Figure 7}

Schematic illustration of PPQDs' protective effect in I/R and potential signaling pathway. $S$ 